
\title{
25 Research Suare \\ Genotype and Clinical Phenotype Analysis of a Family of Kennedy Disease
}

CAl benchi

Hainan Medical University https://orcid.org/0000-0003-4235-1127

\section{Zhiyan SUI}

Hainan Medical University

Tao Chen ( $\nabla$ ctxwxyc@163.com )

Hainan Medical University

\section{Research}

Keywords: Kennedy's disease, gene analysis, clinical phenotype, family analysis

Posted Date: October 28th, 2020

DOI: https://doi.org/10.21203/rs.3.rs-96775/v1

License: (c) This work is licensed under a Creative Commons Attribution 4.0 International License. Read Full License 


\section{Abstract}

Objective: By analyzing the clinical phenotype, genotype and family characteristics of a Kennedy disease family, to explore the clinical and genetic characteristics of the family, and to further improve the understanding of the disease. And through the analysis of genotype and clinical phenotype to draw a pedigree diagram, provide genetic counseling, in order to block the continued inheritance of disease-causing genes.

Methods: The research subjects were from a family of Kennedy disease discovered in January 2019. For 5 clinically diagnosed patients including the proband, the Kennedy's disease function rating scale (KD1234) and the spinal cord and bulbar muscular atrophy rating scale (SBMAFRS) were used to assess their condition, and their clinical phenotypes, laboratory tests, and neurological Analysis of electrophysiological results. After the patient's informed consent was obtained, $5 \mathrm{ml}$ of venous blood was drawn from the 3 members of the proband, and the CAG repeat sequence of the first exon of the androgen receptor (AR) gene was checked by polymerase chain reaction (PCR), and genetic testing was performed. analysis.

Results: 1) Clinical features: The average age of onset of 5 clinical diagnosis members was $(30.8 \pm 2.85)$ years, and the average course of disease was $(12.3 \pm 4.93)$ years at the age of onset to milestones. This family has gynecomastia as the first symptom, and it is the most significant clinical feature. The lesions mainly involve the medulla oblongata and spinal cord. The testosterone level of 5 cases was in the normal range, and the progesterone was increased. 3 cases of estradiol were slightly elevated. The serum creatine kinase (CK) was significantly increased in 5 cases. Electromyography showed chronic extensive neurogenic damage, abnormal sensory and motor conduction. 2) The results of genetic testing showed that the 3 clinically diagnosed cases underwent genetic testing for $48 \mathrm{CAG}$ repetitions ( $\geq 35$ times are abnormal), and genetic diagnosis was confirmed, indicating stable inheritance.

Conclusion: 1) Gynecomastia is the first symptom of the family. Androgen resistance symptoms are the outstanding clinical manifestations of this family, in addition to gynecomastia, including testicular atrophy, infertility, sexual dysfunction, etc.; 2) Changes in serum creatine kinase may indicate the progress or relief of symptoms; 3 ) This family There is chronic extensive neurogenic damage, prominent with sensory nerve involvement; 4) Rehabilitation training may delay the progression of muscle wasting symptoms; 5) There is a stable inheritance of CAG repeats in this family; 6) Genetic testing can diagnose the disease, The genotype is consistent with the clinical phenotype; 7) The pedigree diagram drawn according to the clinical phenotype and genotype can be used as an important basis for genetic counseling.

\section{Background}

Kennedy's disease (KD), also known as spinal and bulbar muscular atrophy (SBMA), is an X-linked recessive genetic neuromuscular disease. The disease was first reported by Kennedy et al. ${ }^{[1]}$ in 1968 . It is characterized by chronic progression of proximal limb and bulbar muscle weakness and muscular atrophy, combined with endocrine system disorders. It is characterized by progressive muscle weakness and atrophy involving the medulla oblongata and spinal cord muscles, accompanied by gynecomastia and decreased fertility, usually between 30 and 50 years of age. In 1991, La Spada ${ }^{[2]}$ discovered that the disease is the androgen receptor (AR) gene located on chromosome Xq11-12, and the cytosine-adenine-guanine (CAG) is caused by abnormal amplification of trinucleotide repeats, which belongs to polyglutamine (polyQ) disease. AR amplified by polyQ 
accumulates abnormally in the nucleus and triggers degeneration of motor neurons and ganglia. Although this disease has always been considered as a pure lower motor neuron disease, the myopathy changes and hypercreatine kinase (CK)emia in muscle biopsy indicate that the existence of primary myopathy ${ }^{[3]}$ cannot be ruled out. The diagnosis of the disease is based on gene sequencing, in which CAG repeats $\geq 35$ are considered pathogenic and recommended as the gold standard for diagnosis ${ }^{[4]}$ by the European guidelines in 2011 . Specific clinical manifestations and auxiliary examinations can also be diagnosed .

The clinical manifestations of Kennedy's disease have certain commonalities, but more studies have suggested that the clinical phenotype of SBMA is obviously heterogeneous, and external factors such as exercise have a great influence on the clinical phenotype of patients ${ }^{[5-8]}$. In addition, although there is no evidence of cardiomyopathy ${ }^{[9]}$ in SBMA patients, other electrocardiogram (ECG) abnormalities may occur, if not detected, it may lead to sudden death ${ }^{[8]}$, which suggests that the disease may involve myocardium. Unique symptoms may also be associated with a high degree of CAG repeat expansion ${ }^{[10]}$. In contrast to the Brazilian and Chilean scholars who reported the country's first patient in recent years, Chinese scholars are at the forefront of the understanding of Kennedy's disease. Due to the large differences in clinical phenotypes between individuals, there are certain controversies. The disease depends on genetic diagnosis. We conducted a clinical phenotype and genotype analysis of a case of Kennedy's disease found in Hainan in 2019 to improve our understanding of the disease.

This study diagnosed the proband through genetic analysis. Using the proband as a clue, other patients in the family were diagnosed through clinical phenotypic analysis, supplemented by genetic analysis. Taking the confirmed patients as clues, suspicious patients and female carriers were screened out through family analysis, and then suspicious members were excluded through genetic analysis, and pre-clinical patients were screened out. Through the analysis of genotype and clinical phenotype, draw a pedigree diagram, and then guide the marriage and childbirth of family members to prevent the continuous inheritance of disease-causing genes.

\section{Methods}

\subsection{Research object}

The study subjects were all from a Kennedy family discovered in 2019. This study and the genetic testing involved were approved by the Ethics Committee of Hainan Provincial People's Hospital. The participants signed an informed consent form, and all subjects received a detailed explanation of this study. And proper genetic counseling. The patient's symptoms were described by the patient and his long-term first-degree relatives, and the signs were evaluated by two professional neurologists. Milestone age is defined as the consensus of patients and family members that it significantly affects the ability of daily living.

\subsection{Laboratory and neuroelectrophysiological examination}

We have perfected serum creatine kinase (CK), serum creatine kinase isoenzyme (CK-MB), biochemical complete set, thyroid seven items, seven gonadal items, electrocardiogram, heart color Doppler ultrasound, etc. Electrophysiological testing is performed using standard nerve conduction techniques and Medelec-Oxford electromyography equipment. 


\subsection{Condition assessment}

KD1234 ${ }^{[5]}$ and the Spinal and Barbar Muscular Atrophy Rating Scale (SBMAFRS) ${ }^{[6]}$ were used for disease assessment. KD1234 includes five aspects: medulla oblongata, upper limbs, lower limbs, and breathing. The full score is 30 points, and the total loss of function is scored as 0 points. SBMAFRS includes medulla oblongata, upper limbs, lower limbs, breathing, and overall functions. The full score is 56 as normal, and the total loss is scored as 0 .

\subsection{Genetic testing}

On the premise of obtaining the patient's informed consent, $5 \mathrm{ml}$ of peripheral venous blood sample of the subject is drawn. The genomic DNA was extracted as a polymerase chain reaction (PCR) template by conventional

methods. According to the primer sequence designed by La Spada ${ }^{[2]}$, the CAG repeat fragment of the first exon of AR gene was amplified by PCR. The primer sequence is: sense strand: 5'-TCCAGAATCTGTTCCAGAGCGTGC-3', antisense strand: 5'-ATGAGGAACAGCAACC TTCACAGC-3', using ActiTaq PCR enzyme system and FAM fluorescently labeled primers for PCR amplification, the conditions are as follows: $95^{\circ} \mathrm{C}$ After denaturation for 90 $\mathrm{s}$, denaturation at $94^{\circ} \mathrm{C}$ for $30 \mathrm{~s}$, annealing at $55^{\circ} \mathrm{C}$ for $30 \mathrm{~s}$, extension at $72^{\circ} \mathrm{C}$ for $60 \mathrm{~s}, 35$ cycles were completed; extension at $72^{\circ} \mathrm{C}$ for $5 \mathrm{~min}$, and kept at $4^{\circ} \mathrm{C}$. The amplified products were subjected to capillary electrophoresis.

\section{Results}

\subsection{General clinical data}

A proband (II-3), 70 years old, male, a farmer by profession, living in Chengmai County, and seeing a doctor in 2019 due to "weak limbs for more than 20 years". Medical history: The patient developed gynecomastia gradually when he was 35 years old and was not paid attention to. At the age of 50 , there is no obvious cause for weakness of the upper limbs, and other androgen resistance symptoms such as testicular atrophy and obvious loss of libido gradually appear. , There are spinal muscle symptoms such as limb girdle muscle weakness, atrophy, fasciculation, etc., and we are admitted to our department as "Kennedy's disease waiting to be discharged". Past history: a history of "lacunar infarction, hyperesteremia" for several years. Family history: The patient's brother and nephew have similar symptoms. Marriage and childbirth history: 1 child at the age of 24, 1 daughter at the age of 26,1 child at the age of 30 , and 1 child at the age of 33. Personal history: average labor intensity, no fixed sports hobbies. Physical examination: high-level nerve activity is normal, cranial nerves: tongue muscle, facial muscle atrophy, tremor, mandibular reflex is positive, and pharyngeal reflex disappears. Motor system: muscle atrophy, fasciculation (interosseous muscles, biceps, quadriceps, supraspinatus, infraspinatus, iliopsoas muscles), muscle strength of the proximal extremities level 3, gait showing duck step. Sensory system: Shallow sensation of both lower limbs is decreased. Reflex: Weakened tendon reflex in limbs, positive bilateral Pap sign. Other: gynecomastia, testicular atrophy, erectile dysfunction. KD1234 scored 16 points, and the medulla oblongata scored 3 points. The SBMAFRS score was 33 points, and the medulla oblongata score was 9 points. This hospitalization was diagnosed with aspiration pneumonia.

II-7, 61 years old, male, occupation is a worker, living in Chengmai County, had been seen in March 2011 due to "episodic memory decline for 14 months" and our department was diagnosed as "1. Episodic memory decline 
checkup" Cause: TIA?, epilepsy? 2. Examination of limb tremor: essential tremor". Medical history: At the age of 33 , the patient developed tremor of both upper limbs, which became worse when he was tense, and gradually found that the breasts were enlarged and libido decreased. Weakness of the limbs gradually appeared around the age of 50 , manifested as unstable walking, squatting and unable to stand, accompanied by perioral and tongue muscle fasciculation, which has gradually increased in recent years. Past history: a history of "diabetes, hypertension". Marriage and childbirth history: 1 daughter at the age of 25 and 1 child at the age of 30 . Personal history: average labor intensity, no fixed sports hobbies. Physical examination: high-level nerve activity is normal, cranial nerve: perioral tremor of the tongue muscle, weakened pharyngeal reflex. Movement system: Muscle atrophy of limbs is obvious, fasciculation is occasionally at the proximal end, muscle strength of limbs is grade 4, gait is duck step. Other: gynecomastia, testicular atrophy, erectile dysfunction. KD1234 scored 22 points, and the medulla oblongata scored 4 points. The SBMAFRS score was 45 points, and the medulla oblongata score was 12 points.

II-5, 65 years old, male, occupation is a demobilized soldier, living in Haikou City. Medical history: At the age of 29, the patient developed upper extremity tremor, which was aggravated during tension and exercise. At the same time, it was found that the breasts developed, the limbs were obviously free of muscle atrophy and fasciculation, and there was no erectile disorder. The patient stated that the ability of daily living was not affected. Personal history: The labor intensity is average, and the patient likes long-distance running, Tai Chi, and martial arts boxing, about 5 times a week, lasting for decades. Past history, marriage and childbirth history is nothing special. Physical examination: high-level nerve activity is normal, cranial nerve: tremor and atrophy of the tongue muscle. Movement system: no muscle atrophy, fasciculation, muscle strength of limbs 5, normal gait. Other:

gynecomastia. KD1234 scored 28 points, and the medulla oblongata scored 5 points. The SBMAFRS score was 51 points, and the medulla oblongata score was 19 points.

III-7, 49 years old, male, occupational worker, living in Haikou City, was admitted to our department in April 2016 due to "repeated limb weakness for more than 10 years, aggravated by 1 week" and diagnosed as "motor neuron disease". Medical history: The patient developed gynecomastia around the age of 30, and gradually felt decreased libido and erectile dysfunction without formal treatment. At the age of 35 , there was no obvious cause of weakness in the limbs, difficulty in raising the hands for a long time, and gradually aggravated the inability to stand up after squatting, accompanied by atrophy of the thenar muscles of the hands, atrophy of the tongue muscles, occasionally choking on drinking water, and unclear articulation. No special treatment. The past history is nothing special. Marriage and childbirth history: gave birth to a daughter when he was 24 years old, and then he failed to take contraceptive measures for more than two years when he wanted to give birth. Personal history: average labor intensity, like walking after dinner, 4 times a week, lasting several years. Physical examination: high-level nerve activity is normal, cranial nerve: perioral tremor and atrophy of the tongue muscle, and loss of pharyngeal reflex. Movement system: the limb girdle muscle atrophy is obvious, no bundle tremor, the proximal muscle strength of the limbs is 4 , the distal muscle strength is 5 , and the gait is duck step. Other: gynecomastia. KD1234 scored 22 points, and the medulla oblongata scored 5 points. The SBMAFRS score was 49 points, and the medulla oblongata score was 14 points.

IV-6, 29 years old, male, occupation is a company employee, living in Haikou City. At the age of 27, there was gynecomastia, no decreased libido, erectile dysfunction, muscle atrophy and fasciculation. The patient stated that the ability of daily living was not affected. Personal history: average labor intensity, no fixed sports hobbies. Physical examination: There is no special physical examination of the nervous system, KD1234 score 30 points,

Page 5/21 
medulla oblongata score 6 points. The SBMAFRS score was 55 points, and the medulla oblongata score was 20 points.

Figure 1 shows the gynecomastia performance of II-3, II-5, III-1, IV-6 from left to right. Four patients agreed to authorize the photos.

The average age of onset of the 5 clinically diagnosed patients was $30.8 \pm 2.85$ years, and the average course of disease was $12.3 \pm 4.93$ years.

The common clinical phenotype of this family is as follows: the first clinical symptom is gynecomastia, and the symptoms of androgen resistance are obvious. In addition to gynecomastia, it also includes testicular atrophy and erectile dysfunction. There are obvious bulbar palsy and fasciculation, the muscle strength of the limbs decreases, and the proximal end is heavier than the distal end, which gradually progresses. Outstanding clinical characteristics of individuals in this family: II-5 has a 36-year course of disease, but apart from gynecomastia, only tongue muscle fasciculation and dysarthria, consider their special personal history and professional background (loving sports, veterans), The amount of exercise has significant characteristics with other individuals, suggesting that exercise may delay the progress of muscle atrophy. 
Table 1

General information of 5 clinically diagnosed patients

\begin{tabular}{|c|c|c|c|c|c|}
\hline Features & II-3 & II-7 & III-7 & IV-6 & II-5 \\
\hline $\begin{array}{l}\text { Age at } \\
\text { diagnosis } \\
\text { (years) }\end{array}$ & 70 & 61 & 49 & 29 & 65 \\
\hline $\begin{array}{l}\text { Age of onset } \\
\text { (years) }\end{array}$ & 35 & 33 & 30 & 27 & 29 \\
\hline $\begin{array}{l}\text { Milestone age } \\
\text { (years) }\end{array}$ & 50 & 50 & 35 & - & - \\
\hline $\begin{array}{l}\text { First } \\
\text { symptoms }\end{array}$ & Gynecomastia & $\begin{array}{l}\text { Gynecomastia } \\
\text { Tremor }\end{array}$ & Gynecomastia & Gynecomastia & Gynecomastia \\
\hline $\begin{array}{l}\text { Sports hobby } \\
\text { (frequency) }\end{array}$ & - & - & $\begin{array}{l}\text { Walking ( } 4 \text { times a } \\
\text { week) }\end{array}$ & - & $\begin{array}{l}\text { Long-distance } \\
\text { running, etc. } \\
\text { ( } 5 \text { times a } \\
\text { week) }\end{array}$ \\
\hline \multicolumn{6}{|c|}{ 1. Muscle fasciculation } \\
\hline surface & + & + & + & - & - \\
\hline Mouth week & + & + & + & - & - \\
\hline $\begin{array}{l}\text { Tongue } \\
\text { muscle }\end{array}$ & + & + & + & - & + \\
\hline Limb Girdle & + & - & - & - & - \\
\hline \multicolumn{6}{|c|}{ 2. Symptoms of bulbar involvement } \\
\hline Dysarthria & + & + & + & - & + \\
\hline Dysphagia & + & + & + & - & - \\
\hline \multicolumn{6}{|c|}{ 3. Androgen resistance symptoms } \\
\hline Gynecomastia & + & + & + & + & + \\
\hline $\begin{array}{l}\text { Erectile } \\
\text { dysfunction }\end{array}$ & + & + & + & - & + \\
\hline $\begin{array}{l}\text { Testicular } \\
\text { atrophy }\end{array}$ & + & + & - & - & - \\
\hline \multicolumn{6}{|c|}{ 4. Muscle strength } \\
\hline $\begin{array}{l}\text { Proximal } \\
\text { Lower } \\
\text { Extremity } \\
\text { (LLR) }\end{array}$ & $3 / 3$ & $4 / 4$ & $4 / 4$ & $5 / 5$ & $5 / 5$ \\
\hline $\begin{array}{l}\text { Distal lower } \\
\text { extremity } \\
\text { (LLR) }\end{array}$ & $4 / 4$ & $4 / 4$ & $5 / 5$ & $5 / 5$ & $5 / 5$ \\
\hline $\begin{array}{l}\text { Proximal } \\
\text { upper }\end{array}$ & $3 / 3$ & $4 / 4$ & $4 / 4$ & $5 / 5$ & $5 / 5$ \\
\hline
\end{tabular}




\begin{tabular}{lccccc}
$\begin{array}{l}\text { Distal upper } \\
\text { limb (LLR) }\end{array}$ & $4 / 4$ & $4 / 4$ & $5 / 5$ & $5 / 5$ & $5 / 5$ \\
\hline $\begin{array}{l}5 . \mathrm{KD} 1234 \\
\text { score }\end{array}$ & 16 & 22 & 22 & 30 & 28 \\
\hline $\begin{array}{l}\text { 6.SBMAFRS } \\
\text { score }\end{array}$ & 33 & 45 & 49 & 55 & 51 \\
\hline
\end{tabular}

\subsection{Laboratory results}

The 5 clinically confirmed patients have perfected laboratory examinations, and the CK and CK-MB before and after II-3 admission are constantly changing: (2019-1-5) 465.5uul, 29.7uul, (2019-1-12) 230.8uul, 21.8 uul, (20191-20)126.3uul, 20.7uul, their KD1234 scores are (2019-1-5) 16 points, (2019-1-12) 19 points, (2019-1-20) 20 points, respectively. CK and CK-MB normal reference range ((CK:50-310uul CK-MB:0-24uul).

Other laboratory results: 5 clinically diagnosed patients with normal thyroid function; electrocardiogram and cardiac color Doppler ultrasound were normal; 2 of them had different degrees of dyslipidemia; 1 had a history of diabetes and was regularly taking metformin to lower blood sugar; 5 patients had testosterone In the normal range (6.49-32.95nmolll before 50 years old and 6.19-34.21 nmolll after 50 years old), progesterone was increased (<0.318-0.636nmollI), and estradiol was slightly increased in 3 cases (40.4-161.5pmollI). See Table 3.2 for details

Table 2

Laboratory test results of 5 clinically diagnosed patients

\begin{tabular}{|llllll|}
\hline Laboratory testing & II-3 & II-7 & III-7 & IV-6 & II-5 \\
\hline Serum Creatine Kinase (CK) & $465.6 \mathrm{U} / \mathrm{L}$ & $645.1 \mathrm{U} / \mathrm{L}$ & $869.9 \mathrm{U} / \mathrm{L}$ & $147.9 \mathrm{u} / \mathrm{I}$ & $149.5 \mathrm{u} / \mathrm{I}$ \\
\hline $\begin{array}{l}\text { Creatine Kinase Isoenzyme } \\
\text { CK-MB) }\end{array}$ & $29.7 \mathrm{U} / \mathrm{L}$ & $30.7 \mathrm{U} / \mathrm{L}$ & $27.0 \mathrm{U} / \mathrm{L}$ & $16.6 \mathrm{u} / \mathrm{I}$ & $15.1 \mathrm{u} / \mathrm{I}$ \\
\hline Triglycerides & $2.30 \mathrm{mmol} / \mathrm{I}$ & $1.49 \mathrm{mmol} / \mathrm{I}$ & $1.03 \mathrm{mmol} / \mathrm{I}$ & $3.54 \mathrm{mmol} / \mathrm{I}$ & $1.37 \mathrm{mmol} / \mathrm{I}$ \\
\hline Serum cholesterol & $4.40 \mathrm{mmol} / \mathrm{I}$ & $3.64 \mathrm{mmol} / \mathrm{I}$ & $3.01 \mathrm{mmol} / \mathrm{I}$ & $5.34 \mathrm{mmol} / \mathrm{I}$ & $3.51 \mathrm{mmol} / \mathrm{I}$ \\
\hline Testosterone & 18.71 & $18.06 \mathrm{nmol} / \mathrm{L}$ & $19.11 \mathrm{nmol} / \mathrm{I}$ & $28.34 \mathrm{nmol} / \mathrm{I}$ & $20.03 \mathrm{nmol} / \mathrm{I}$ \\
& $\mathrm{nmol} / \mathrm{L}$ & & & & \\
\hline Progesterone & $0.98 \mathrm{nmol} / \mathrm{L}$ & $0.91 \mathrm{nmol} / \mathrm{L}$ & $0.87 \mathrm{nmol} / \mathrm{L}$ & $0.71 \mathrm{nmol} / \mathrm{I}$ & $0.83 \mathrm{nmol} / \mathrm{L}$ \\
\hline Estradiol & $153.0 \mathrm{pmol} / \mathrm{L}$ & $43.0 \mathrm{pmol} / \mathrm{L}$ & $163.0 \mathrm{pmol} / \mathrm{L}$ & $226.0 \mathrm{pmol} / \mathrm{I}$ & $\begin{array}{l}170.0 \\
\mathrm{pmol} / \mathrm{L}\end{array}$ \\
\hline
\end{tabular}

\subsection{Electrophysiological results}


The electrophysiological examinations were perfected in the 5 clinically diagnosed patients except for the rejection of II-5. Among them, the muscles examined in II-3, II-7, and III-7 showed chronic and extensive neurogenic damage on the electromyogram, mainly involving the limbs Muscles, sternocleidomastoid muscle, thoracic paraspinal muscles, abdominal muscles, tongue muscles, orbicularis orbicularis muscles, of which III-7 is due to multiple waves, and the possibility of myogenic damage cannot be ruled out. IV- 6 concentric needles The electrode inspection results showed no obvious abnormalities. The compound action potential of the peripheral nerves examined in the extremities has low amplitude, slowed down the conduction speed, and the incubation period is roughly normal. The distal sensory nerve has significantly low amplitude and slowed conduction speed, of which II-3 and II-7 are the most significant. See Table 3 for details 
Table 3

Electrophysiological results of clinically confirmed patients

\begin{tabular}{|c|c|c|c|c|}
\hline Neurophysiology & II-3 & $\|-7$ & III-7 & IV-6 \\
\hline \multicolumn{5}{|l|}{ Median } \\
\hline $\mathrm{CMAP}(\mathrm{mv})$ & 5.4 & 3.4 & 6.4 & 15.2 \\
\hline $\operatorname{MCV}(\mathrm{m} / \mathrm{s})$ & 47.8 & 53.4 & 62.1 & 56.4 \\
\hline $\mathrm{DL}(\mathrm{ms})$ & 4.8 & 3.3 & 3.8 & 3.1 \\
\hline \multicolumn{5}{|l|}{ Ulnar nerve (Ulnar) } \\
\hline CMAP(mv) & 6.1 & 7.8 & 3.8 & 11.1 \\
\hline $\operatorname{MCV}(\mathrm{m} / \mathrm{s})$ & 49.0 & 57.1 & 57.4 & 53.3 \\
\hline $\mathrm{DL}(\mathrm{ms})$ & 3.9 & 2.9 & 3.4 & 2.7 \\
\hline \multicolumn{5}{|c|}{ Tibial nerve (Tibial) } \\
\hline CMAP(mv) & 12.8 & 18.3 & 13.7 & 18.2 \\
\hline $\operatorname{MCV}(\mathrm{m} / \mathrm{s})$ & 43.6 & 52.6 & 50.0 & 44.0 \\
\hline $\mathrm{DL}(\mathrm{ms})$ & 5.3 & 4.4 & 5.0 & 3.5 \\
\hline \multicolumn{5}{|c|}{ Common peroneal nerve (peroneal) } \\
\hline CMAP(mv) & 7.5 & 1.0 & 5.5 & 11.1 \\
\hline $\operatorname{MCV}(\mathrm{m} / \mathrm{s})$ & 44.9 & 48.3 & 48.5 & 49.1 \\
\hline $\mathrm{DL}(\mathrm{ms})$ & 3.6 & 5.6 & 4.7 & 3.9 \\
\hline \multicolumn{5}{|l|}{ Median } \\
\hline SNAP『uv》 & 4.2 & 6.0 & 49.0 & 38.0 \\
\hline $\mathrm{SCV} \otimes \mathrm{m} / \mathrm{s} \rrbracket$ & 48.1 & 50.0 & 60.0 & 57.1 \\
\hline \multicolumn{5}{|l|}{ Ulnar nerve (Ulnar) } \\
\hline SNAP\uv》 & 3.3 & 3.1 & 19.0 & 25.0 \\
\hline $\mathrm{SCV} \otimes \mathrm{m} / \mathrm{s} \rrbracket$ & 56.4 & 56.6 & 53.0 & 50.5 \\
\hline \multicolumn{5}{|l|}{ Sural nerve (sural) } \\
\hline SNAP』uv凹 & 4.5 & 4.4 & 10.0 & 20.0 \\
\hline$S C V \otimes m / s \rrbracket$ & 47.0 & 47.2 & 44.0 & 54.5 \\
\hline
\end{tabular}

\subsection{Genetic test results}

Three of the five clinically diagnosed patients agreed to undergo genetic testing. Among them, the number of CAG repetitions of the proband (II-3) is 48 , and the number of repetitions of the three generations of II-3 (Figure 
2.1), III-7 (Figure 2.2), and IV-6 (Figure 2.3) is 48. Consider The number of CAG repeats is stably inherited in this family. (See Figure 2).

\subsection{Genealogical analysis results}

Comprehensive clinical phenotype and genetic testing, the proband II-3 is clearly diagnosed as KD patients, and through clinical phenotype analysis II-5, II-7, III-7, and IV-6 are all clinically diagnosed patients, and genotype analysis (II-5, II-7 refused), both showed that the number of CAG repeats was the same as that of the proband, 48 times, showing stable inheritance. It is inferred that II-5 and II-7 are also confirmed patients. Based on the analysis of 5 confirmed patients and the X-linked recessive genetic characteristics of this disease, it is speculated that: I-1, II-1, III-3, III-13, III-21, IV-9 are female carrier members, III-11 , III-16, III-18, III-20, III-23, IV-3, IV-4, IV-10, IV-11 are normal men, IV-5, IV-6, IV-7, IV- 17. IV-22 is a suspicious member, and III-5, IV-1, IV-2, IV-8, V-13, IV-14, IV-15, IV16 are suspicious female carriers. Draw a pedigree diagram to provide a basis for genetic counseling of the family (Figure 3.5).

\section{Discussion}

\section{1 clinical phenotype analysis}

Progressive proximal spinal cord and bulbar muscular atrophy (SBMA) was first described by William R. Kennedy et al. in 1968, and then 2 families were examined, of which 11 members (all men) developed delayed slow progressive neuromuscular Disease ${ }^{[1]}$. Decades later, the genetic basis of SBMA was determined as the expansion of the polymorphic tandem CAG repeat sequence ${ }^{[2}$ in the first exon of the androgen receptor (AR) gene. SBMA, the first known CAG trinucleotide repetitive disease, currently exists in types $1,2,3,6,7$, and 17 including Huntington's disease, dentate nucleus globular atrophy and spinocerebellar ataxia. Spinal cord and bulbar muscular atrophy (SBMA) is an X-linked recessive polyQ disease. The latest epidemiological study from Italy suggests that the prevalence is about $2.588100000^{[15]}$. Kennedy's disease usually occurs in young and middle-aged men. It can manifest as weakness of the proximal lower limbs, and the annual muscle strength decreases by about $2 \%{ }^{[16]}$. Other symptoms appear over time, including tremor, muscle spasm, muscle fasciculation, and dysarthria And dysphagia ${ }^{[17]}$.

The pathological features of Kennedy's disease are the loss of lower motor neurons in the anterior horn of the spinal cord and brain stem ${ }^{[18]}$, and signs of myogenic cytotoxicity ${ }^{[19-21]}$. Some studies believe that the best characteristic metabolic index of SBMA is serum creatine kinase (CK). In most SBMA patients, it rises to about 34 times the normal range ${ }^{[22,23]}$, which is higher than that of pure neurogenicity. The expectation of the disease emphasizes the fact that primary myopathy causes SBMA ${ }^{[21]}$. In this family II-3, II-7, III-7 serum CK was significantly increased. The changes of CK during II-3 hospitalization and the gradual relief of patients' muscle symptoms suggest that CK is a sign of active or worsening muscle symptoms. At the same time, studies $^{[3]}$ support that CK is related to disease severity, confirm its role as a valuable biomarker in SBMA, and highlight the relevant role of skeletal muscle in disease pathogenesis. 
As the course of the disease gradually increased, the scores of KD1234 and SMBAFRS decreased, but the longest course score of II-5 was only lower than that of IV-6, which suggests that maintaining a certain intensity of exercise may delay the progression of the disease, which is similar to Karen ${ }^{[24]}$ The same point of view. It is well known that exercise is beneficial to health in general, but caution should be used when using exercise as a treatment for neuromuscular diseases, because there is a view that exercise may increase the rate of muscle wasting in patients with ALS. This is mainly based on two observations: firstly, high-level athletes have a greater risk of $A L S^{[25]}$; secondly, when the onset of symptoms in ALS patients is asymmetrical, major lateral muscle weakness accounts for about $70 \%$ of the main complaints ${ }^{[26]}$. So far, in clinical trials studying the effects of exercise on ALS patients, these problems have been noted, but no negative outcomes have been reported. In fact, studies have shown that exercise can significantly improve the muscle strength ${ }^{[27]}$ and quality of life score ${ }^{[28]}$ of patients with ALS. There is another basis for the therapeutic potential of exercise therapy for SBMA, because exercise is known to increase the circulating levels of insulin-like growth factor $1\left(\right.$ IGF-1 ${ }^{[29]}$, which has a protective effect in SBMA animal models ${ }^{[30]}$. Based on these data, a clinical study was conducted in which 50 SBMA patients participated in 12 weeks of "functional exercise" (24 patients) or stretching (control, 26 patients) ${ }^{\text {[ }}$ 31]. Overall, exercise in the primary outcome measure (Adult Myopathy Assessment Tool (AMAT) score) or any of the secondary measures (including muscle strength, balance, quality of life score, and IGF-1 level assessment) did not result in significant improvement ${ }^{[32]}$. However, when the patients were divided into high or low function groups according to the initial AMAT score, it was found that exercise significantly improved the AMAT score of low function patients ${ }^{[33]}$. These results may indicate that the functional exercise routine performed by patients in this study is too light, especially for patients who are subdivided into high-functioning groups. The effect of highintensity training on the progression of SBMA disease is currently being studied (clinicaltrials.govNCT02156141).

Some recent studies suggest that impaired glucose homeostasis may be a common feature of SBMA ${ }^{[23,25,34]}$. Although the results of these studies have conflicting evidence on fasting blood glucose, the homeostatic model assessment of insulin resistance ${ }^{[34,35]}$ seems to have reached a consensus on the increase in insulin resistance in SBMA patients, and found that insulin resistance and insulin resistance in the SBMA patient cohort There is a significant correlation between motor dysfunction, but the mechanism of insulin resistance in SBMA patients needs to be further clarified ${ }^{[34]}$. Nevertheless, these results indicate that the symptoms of metabolic disorders in SBMA patients may be an effective tool for assessing the condition. Other metabolic index disorders were reported in the subgroup of SBMA patients, including low body mass index, high blood pressure, total cholesterol and triglycerides ${ }^{[34,35]}$. Both II-3 and IV-6 in this family have different degrees of dyslipidemia. II-5 has previously confirmed diabetes, which does not violate the above conclusion. Giorgia ${ }^{[36]}$ and others recently designed a "6-K scale" to refine the score of cranial nerve innervation muscles, but still did not add non-motor symptoms. Some studies have found that SBMA patients have decreased working memory and executive function ${ }^{[37,38]}$. In addition, a neuropsychological analysis of 20 SBMA patients found a mental deficit, which was confirmed by the performance of the Faux Pas test ${ }^{[39]}$. These results indicate that the frontal lobe is involved in the pathogenesis of SBMA, which further supports the fact that SBMA patients have insufficient glucose metabolism ${ }^{[40]}{ }_{a n d}$ extensive atrophy of the frontal white matter of the brain ${ }^{[41]}$. These studies seem to explain some of the potential cognitive impairment of II-7. 
Electromyography has received widespread attention as a preliminary screening test for this disease. Among them, $\mathrm{Kim}^{[42]}$ and others retrospectively analyzed 62 cases of SBMA patients, and believed that the CAG repetition size is negatively correlated with the compound muscle action potential (CMAP) amplitude. No significant correlation was found between CAG repeat size and sensory nerve action potential (SNAP) amplitude. CAG repetition size is only negatively correlated with CMAP amplitude, but not negatively correlated with SNAP amplitude. This is contrary to previous reports about the motor and sensory dominant phenotypes related to CAG repetition size. But Hama ${ }^{[43]}$ et al. believe that the specific reduction of SNAP amplitude in SBMA provides another useful tool for the differential diagnosis of motor neuron diseases. The number of CAG repetitions in this family is the same, both are 48 times, which can not confirm the relationship between CAG repetition times and nerve conduction. Electrophysiological results suggest that this family has chronic extensive neurogenic damage, but the III-7 EMG results suggest that the possibility of combined myogenic damage cannot be ruled out. Sensory and motor conduction are abnormal, but sensory neuropathy such as reduced sensory nerve action potential (SNAP) amplitude and slower sensory nerve conduction velocity (SCV) is more common and significant than motor nerve involvement, which is consistent with Hama et al.

\subsection{Genotype analysis}

The clinical phenotypes of this family are similar. The possible reasons are the same CAG repeat sequence, the same characteristics of hormone level changes, and the same genetic background in other families. In terms of disease assessment, neither of the two scales included the symptoms of androgen resistance and metabolic disorders in the evaluation system. This suggests that we need to establish a new evaluation system to include non-motor symptoms, including potential endocrine disorders such as lipid metabolism, glucose metabolism disorders, and androgen resistance symptoms such as testicular atrophy and gynecomastia.

The results of electromyography suggest that this family has chronic extensive neurogenic damage, which is prominent in sensory nerve involvement. Male patients in this family have gynecomastia and appear as the first symptom, so the diagnosis cannot be made based on gynecomastia combined with other clinical phenotypes. However, there are also reports of Kennedy's disease families without bulbar palsy and androgen resistance. Therefore, although androgen insensitivity is a characteristic symptom of SBMA, it is not a necessary condition for diagnosis. When adult men without bulbar palsy symptoms and androgen resistance symptoms develop chronic lower motor neuron paralysis, Kennedy's disease must also be considered.

The gold standard for the diagnosis of this disease is the number of CAG repeats. The number of CAG repeats in the three non-first-degree relatives of the second, third and fourth generations in this family is 48 , indicating that the number of CAG repeats is stable in the family. . The genotype consistency of this family is the same as reported by MacLean ${ }^{[44]}$. In order to avoid the continued inheritance of pathogenic genes, the main methods are: suspicious patients and female carriers detection, prenatal genetic diagnosis and preimplantation genetic diagnosis. For the detection of suspicious patients and female carriers, IV-7 is clinically asymptomatic and Kendy's disease is excluded through genetic diagnosis. The sequence number is 22, while IV-5 and IV-22 are clinically asymptomatic but have not participated in this project to complete the gene The test cannot be diagnosed. It is recommended that the suspected carrier or diseased members of the IV generation who have not given birth to carry out genetic diagnosis as soon as possible; prenatal genetic diagnosis uses amplified gene fragments to quickly and accurately perform gender identification and prenatal diagnosis, so as to identify the 
sex of the fetus and the pathogenic gene carrier Whether or not; preimplantation genetic diagnosis can screen out embryo sex by fluorescent in situ immunoassay of in vitro fertilized eggs, and exclude the occurrence of sexlinked diseases. According to the characteristics of Kennedy's disease X-linked recessive inheritance, the following suggestions are given to members who have not given birth: 1) It is recommended that the wife of IV-6 undergo gender identification before giving birth, and inform the girl born to carry the disease-causing gene, and the girl's offspring There is a 112 probability to continue to carry the disease-causing gene, and the boys who give birth may not carry the disease-causing gene. 2) There is a 112 probability that girls giving birth to IV-9 children will continue to carry the disease-causing gene, and the next generation of boys will still be at risk.

\section{Conclusion}

1) Gynecomastia is the first symptom of the family. Androgen resistance symptoms are the outstanding clinical manifestations of this family, in addition to gynecomastia, including testicular atrophy, infertility, sexual dysfunction, etc.; 2) Changes in serum creatine kinase may indicate the progress or relief of symptoms; 3 ) This family There is chronic extensive neurogenic damage, prominent with sensory nerve involvement; 4)

Rehabilitation training may delay the progression of muscle wasting symptoms; 5) There is a stable inheritance of CAG repeats in this family; 6 ) Genetic testing can diagnose the disease, The genotype is consistent with the clinical phenotype; 7) The pedigree diagram drawn according to the clinical phenotype and genotype can be used as an important basis for genetic counseling.

\section{Abbreviations}

\begin{tabular}{ll} 
KD1234 & Kennedy's disease function rating scale \\
\hline SBMAFRS & Bbulbar muscular atrophy rating scale \\
\hline AR & androgen receptor \\
\hline CAG & cytosine-adenine-guanine \\
\hline AMAT & Adult Myopathy Assessment Tool \\
\hline KD & Kennedy's disease \\
\hline SBMA & spinal and bulbar muscular atrophy
\end{tabular}

\section{Declarations}

\section{Availability of data and materials:}

The datasets used and/or analyzed during the current study are available from the corresponding author on reasonable request.

\section{Ethics approval and consent to participate:}

This study was approved by the Institutional Review Board of the The HAINAN General Medical Center (approved number:799,January 2019). 


\section{Consent for publication}

All study participants provided written, informed consent. \The attachment is the consent of our design.囚

\section{Authors'contributions:}

Cai Benchi designed and performed the experiments and wrote the manuscript; Sui Zhiyan and Chen Tao provided key reagents and advice; Chen Tao provided funding

\section{Funding:}

This work was supported by the Chinese National Natural Science Foundation(81560202)

\section{Competing interests】}

We declare that they have no competing interests.

\section{Acknowledgements:}

The author would like to thank the patients who took part in the research.

\section{References}

1. Kennedy WR, IterM A, Sung JH. Progressive prox imal spinal and bulbarmuscular atrophy of late onset. A sexlinked recessive trait[J]. Neurology. 1968;18:671680.

2. La Spada AR, ilson W, Lubahn EM. DB, et a.I Androgen receptor gene mutation in Xlinked spinal and bulbar muscular atrophy[J]. N ature. 1991;352:7779.

3. Vittoria Lombardi,Giorgia Querin,Oliver J,et al. Muscle and not neuronal biomarkers correlate with severity in spinal and bulbar muscular atrophy[J]. Neurology,2019,92(11).

4. Burgunder JM, Schols L, Baets J,et al. EFNS guidelines for the molecular diagnosis of neurogenetic disorders: motoneuron,peripheral nerve and muscle disorders[J]. Eur J Neurol. 2011;18(2):207-17.

5. Lu M, Guo H, Fan D,et al.Kennedy's disease 1234 scale: Preliminary design and test[J]. Clin Neurosci.2017 Feb 24.

6. Hashizume A, Katsuno M, Suzuki K,et al. A functional scale for spinal and bulbar muscular atrophy:Crosssectional and longitudinal study[J]. Hashizume ANeuromuscul Disord.2015 Jul;25(7):554-62.

7. Romigi A, Liguori C, Placidi F,et al. Sleep disorders in spinal and bulbar muscular atrophy (Kennedy's disease): a controlled polysomnographic and selfreported questionnaires study[J]. Neurol. 2014;261(5):88993.

8. Sumner CJ, Fischbeck KH. Jaw drop in Kennedy's disease[J].Neurology. 2002;59(9):1471-2.

9. Praline J, GuennocAM, Malinge MC,et al. Pure bulbar motor neuron involvement linked to an abnormal CAG repeat expansion in the androgen receptor gene[J]. Amyotroph Lateral Scler. 2008;9(1):40-2. 
10. ArakiK,NakanishiH,NakamuraT,AtsutaN. et al. Myotonia-like symptoms in a patient with spinal and bulbar muscular atrophy[J]. Neuromuscul Disord. 2015;25(11):913-5.

11. Quering,MelaciniP, D'AscenzoC,MorandiL, et al. No evidence of cardiomyopathy in spinal and bulbar muscular atrophy[J]. Acta Neurol Scand. 2013;128(6):e30-2.

12. Grunseich C, Kats IR, Bott LC, Rinaldi C, et al. Early onsetand novel features ina spinal and bulbar muscular atrophy patient with a 68 CAG repeat[J]. Neuromuscul Disord. 2014;24(11):978-81.

13. Mariotti C, Castellotti B, Pareyson D,et al. Phenotypic manifestations associated with CAG-repeat expansion in the androgen receptor gene in male patients and heterozygous females:a clinical and molecular study of 30 families[J]. Neuromuscul Disord. 2000;10:391-7.

14. Lund A, Udd B, Juvonen V,et al.Multiple founder effects in spinal and bulbar muscular around the world[J]. Eur J Hum Genet.2001;9:43 I-436.

15. Bertolin G, Querin I,Martinelli M,et al.Insights into the genetic epidemiology of spinal and bulbar muscular atrophy: prevalence estimation and multiple founder haplotypes in the Veneto Italian region[J].European Journal of Neurology,2019,26(3).

16. Fernández-Rhodes LE, Kokkinis AD, White MJ. et Al.Efficacy and safety of dutasteride in patients with spinal and bulbar muscular atrophy: a randomised placebo-controlled tria[J]. Lancet Neurol. 2011 Feb;10(2):140-7.

17. Atsuta $\mathrm{N}$,Watanabe $\mathrm{H}$,Ito $\mathrm{M}$,et Al.Banno HNatural history of spinal and bulbar muscular atrophy (SBMA): a study of 223 Japanese patients[J]. Brain.2006 Jun;129(Pt 6):1446-55.

18. Amato AA, Prior TW, Barohn RJ. et Al. Kennedy disease: a clinicopathologiccorrelation withmutations in the androgen receptor gene[J]. Neurology. 1993;43(4):791-4.

19. Cortes CJ, Ling SC, Guo LT, Hung G, et al. Muscle expression of mutant androgen receptor accounts for systemic and motor neuron disease phenotypes in spinal and bulbar muscular atrophy[J]. Neuron. 2014;82(2):295-307.

20. BannoH. KatsunoM,SuzukiK,TanakaF,SobueG.Pathogenesis and molecular targeted therapy of spinal and bulbar muscular atrophy (SBMA)[J]. Cell Tissue Res. 2012;349(1):313-20.

21. Sorarù G, Ascenzo C, Polo A,et al. Spinal and bulbar muscular atrophy: skeletal muscle pathology in male patients and heterozygous females[J]. Neurol Sci. 2008;264(1-2):100-5.

22. Chahin N, Sorenson EJ. Serum creatine kinase levels in spinobulbar muscular atrophy and amyotrophic lateral sclerosis[J]. Muscle Nerve. 2009;40(1):126-9. 12.

23. Querin G,Bertolin C,DaRe E,VolpeM,ZaraG,PegoraroE,et al. Non-neural phenotype of spinal and bulbar muscular atrophy: results from a large cohort of Italian patients[J]. Neurol Neurosurg Psychiatry. 2016;87(8):810-6.

24. Karen $\mathrm{H}$,Grete Andersen,Astrid Buch,et al.High-intensity training in patients with spinal and bulbar muscular atrophy[J]. Journal of Neurology,2019,266(7).

25. Gotkine M, Friedlander $Y$, Hochner H. Triathletes are overrepresented in a population of patients with ALS[J]. Amyotroph Lateral Scler Frontotemporal Degener. 2014;15(7-8):534-6.

26. Fratta P, Nirmalananthan N, Masset L, et al. Correlation of clinical and molecular features in spinal bulbar muscular atrophy[J]. Neurology. 2014;82(23):2077-84.

27. Drory VE, Goltsman E, Reznik JG. The valueofmuscle exerciseinpatients with amyotrophic lateralsclerosis[J]. Neurol Sci. 2001;191(1-2):133-7. 
28. Bello-Haas VD, Florence JM, Kloos AD, et al. A randomized controlled trial of resistance exercise in individuals with ALS[J]. Neurology. 2007;68(23):2003-7.

29. Schwarz AJ, Brasel JA, Hintz RL. Acute effect of brief low- and high-intensity exercise on circulating insulinlike growth factor (IGF) I, II, and IGF-binding protein-3 and its proteolysis in young healthy men[J]. Clin Endocrinol Metab. 1996;81(10):3492-7.

30. Rinaldi C, Bott LC, Chen KL, et al. Insulinlike growth factor (IGF)-1 administration ameliorates disease manifestations in a mouse model of spinal and bulbar muscular atrophy[J]. Mol Med. 2012;18:1261-8.

31. Shrader JA, Kats I, Kokkinis A. etal.Arandomized controlled trial of exercise in spinal and bulbar muscular atrophy[J]. Ann Clin TransI Neurol. 2015;2(7):739-47.

32. Dahlqvist JR. VissingJ.Exercise the rapy in bulbar muscular atrophy and other neuromuscular disorders.[J]. Mol Neurosci. 2016;58(3):388-93.

33. Nakatsuji H, Araki A, Hashizume A,et al. Correlation of insulin resistance motor functioninspinalandbulbarmuscularatrophy[J]Neurol. 2017;264(5):839-47.

34. Rosenbohm A, Hirsch S, Volk AE, Grehl T, Grosskreutz J, Hanisch F, et al. The metabolic and endocrine characteristics in spinal and bulbar muscular atrophy[J]. Neurol. 2018;265(5):1026-36.

35. Wang Z, Chen Q, Li Q,et al.Clinical features of a genetically identified spinal and bulbar muscular atrophy pedigree.[J]Zhong Nan Da Xue Xue Bao Yi Xue Ban. 2016 Oct 28.

36. Giorgia Q, Irene B, Laura M,et al.Preliminary design and validation of the "6-K-scale" for bulbar symptoms evaluation in SBMA.[J].Neurol Sci. 2019 Jul;40(7):1393-1401.

37. Soukup GR, Sperfeld AD, Uttner I, et al. Frontotemporal cognitive function in X-linked spinal and bulbar muscular atrophy (SBMA): a controlled neuropsychological study of20patients[J]. Neurol. 2009;256(11):1869-75.

38. Kasper E. Wegrzyn M,Marx I,etal. Minor cognitive disturbances in X-linked spinal and bulbar muscular atrophy, Kennedy's disease[J]. Amyotroph Lateral Scler Frontotemporal Degener. 2014;15(1-2):15-20.

39. Di Rosa E, Sorarù G, Kleinbub JR,et al. Theory of mind, empathy and neuropsychological functioning in Xlinked spinal and bulbar muscular atrophy: a controlled study of 20 patients[J]. Neurol. 2015;262(2):394401.

40. Lai TH, Liu RS, Yang BH, et al. Cerebral involvement in spinal and bulbar muscular atrophy (Kennedy's disease): a pilot study of PET[J]. Neurol Sci. 2013;335(1-2):139-44.

41. Kassube, kJ. Juengling FD,Sperfeld AD.Widespreadwhitematter changes in Kennedy disease: a voxel based morphometry study[J]. Neurol Neurosurg Psychiatry. 2007;78(11):1209-12.

42. $\mathrm{Kim} \mathrm{H}$, et al.Correlation between the CAG repeat size and electrophysiological findings in patients with spinal and bulbar muscular atrophy.J.Muscle Nerve. 2018 Apr;57(4):683-686.

43. Hama T, Hirayama M, Hara T,et al.Discrimination of spinal and bulbar muscular atrophy from amyotrophic lateral sclerosis using sensory nerve action potentials.[J].Muscle Nerve. 2012 Feb;45(2):169-74.

44. MacLean HE, Gonzales M, Greenland KJ,et al. Age-dependent differences in androgen binding affinity in a family with spinal and bulbar muscular atrophy[J]. Neurol Res. 2005 Jul;27(5):548-51.

\section{Figures}




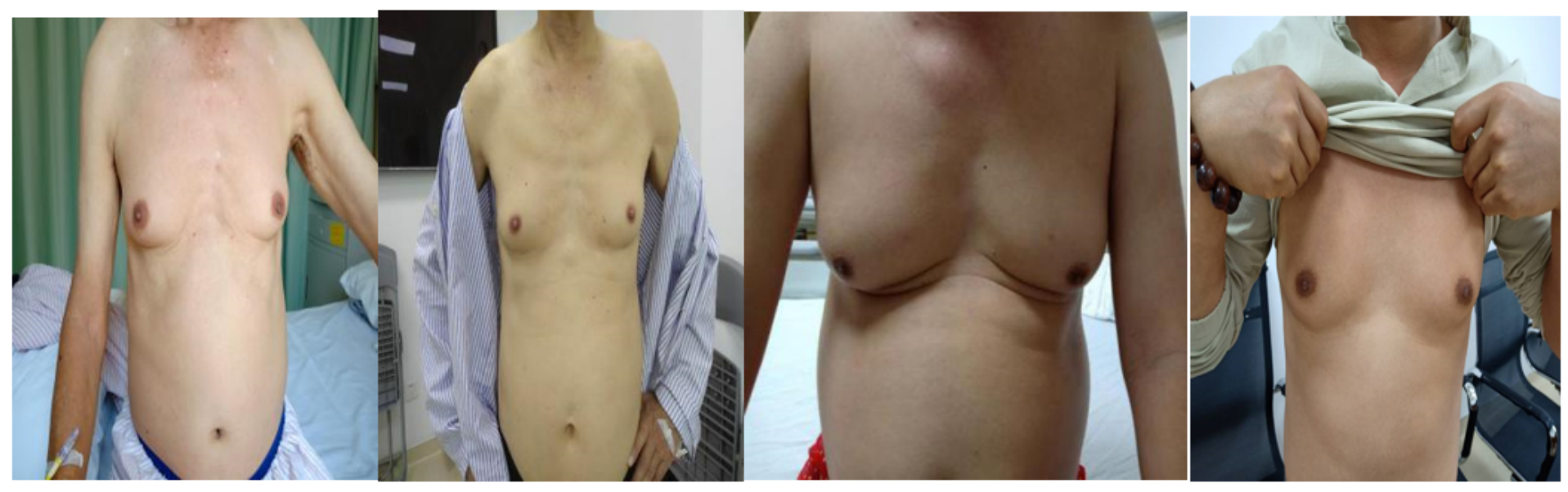

Figure 1

shows the gynecomastia performance of II-3, II-5, III-1, IV-6 from left to right. Four patients agreed to authorize the photos.

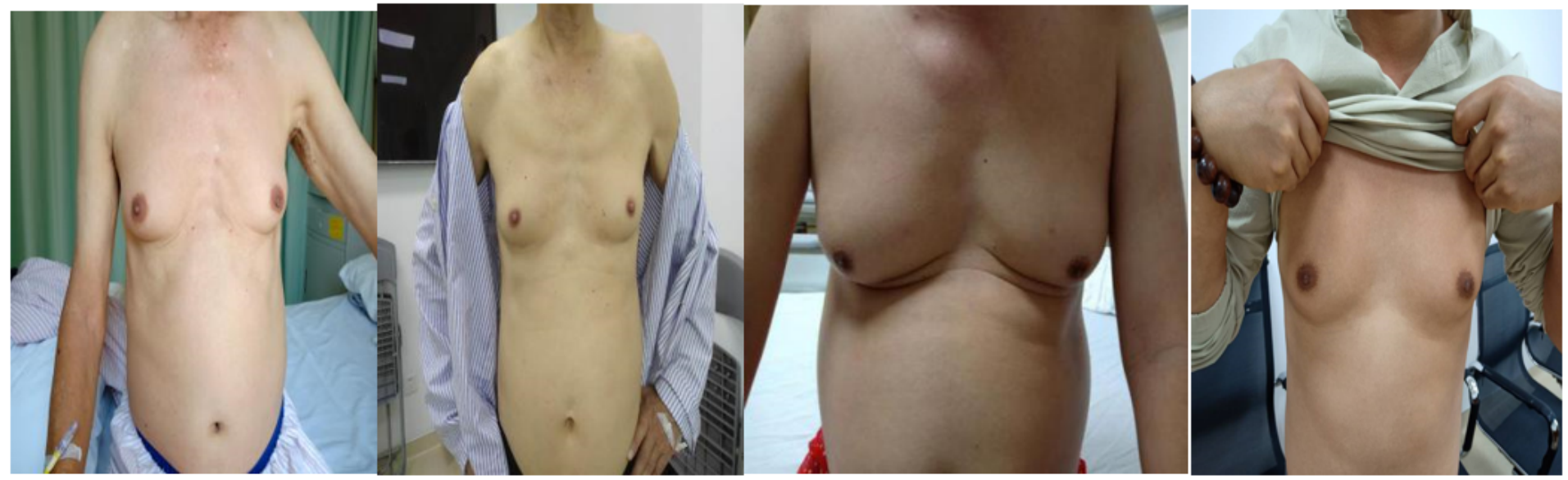

Figure 1

shows the gynecomastia performance of II-3, II-5, III-1, IV-6 from left to right. Four patients agreed to authorize the photos. 
$(2.1)$ AR-CP21D0270(6335) 014 2019-03-01 B02.fsa AR-CP21D0270(6335) None

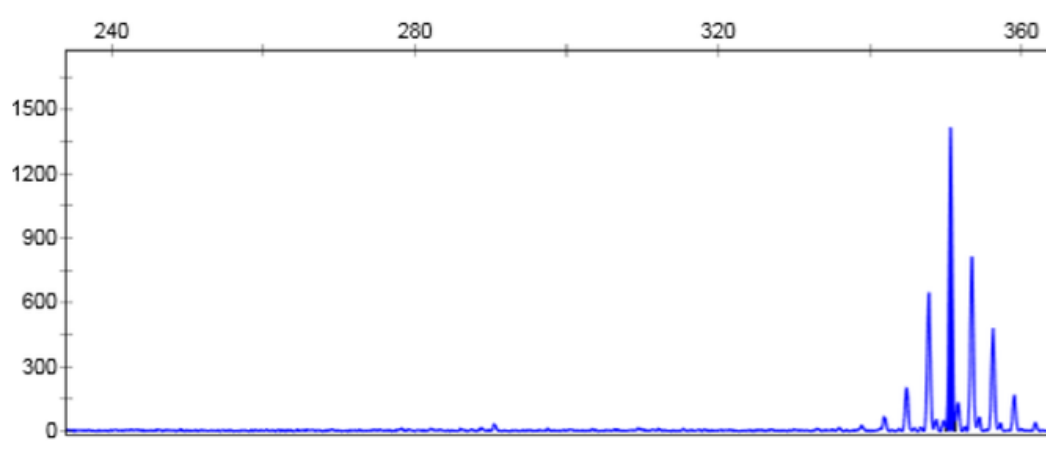

(2.2)
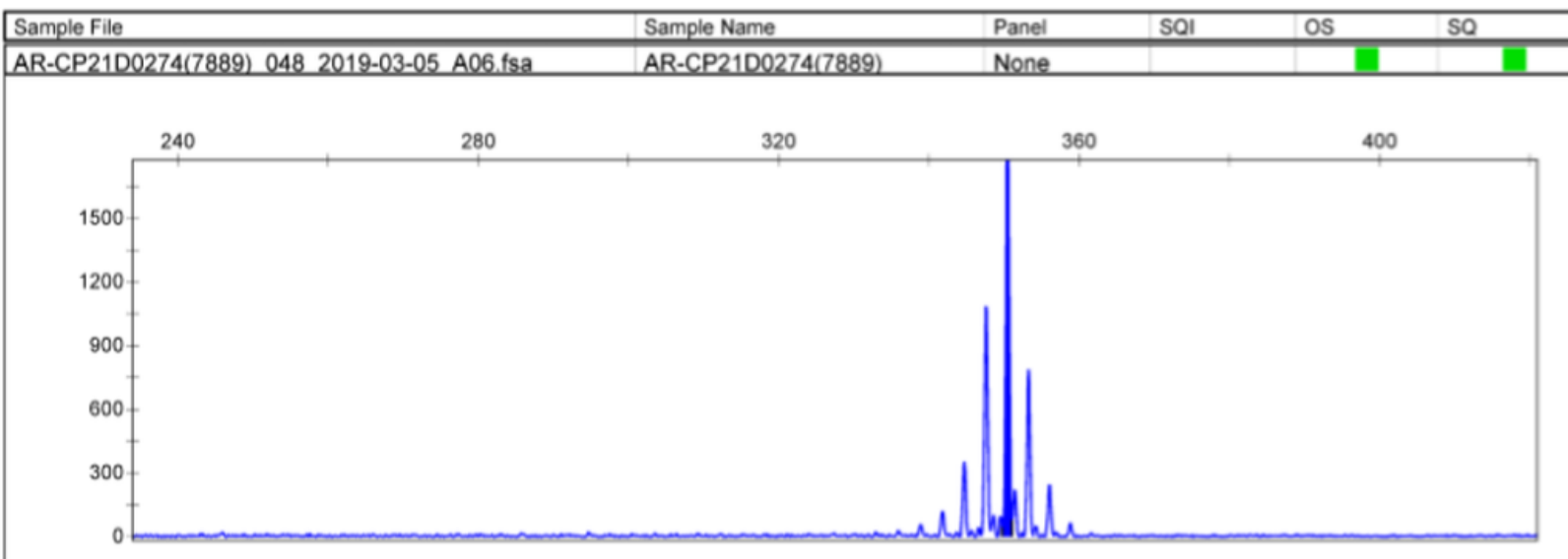

(2.3)

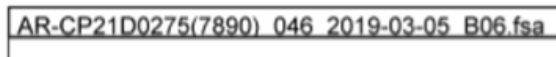
AR-CP2100275(7890) None

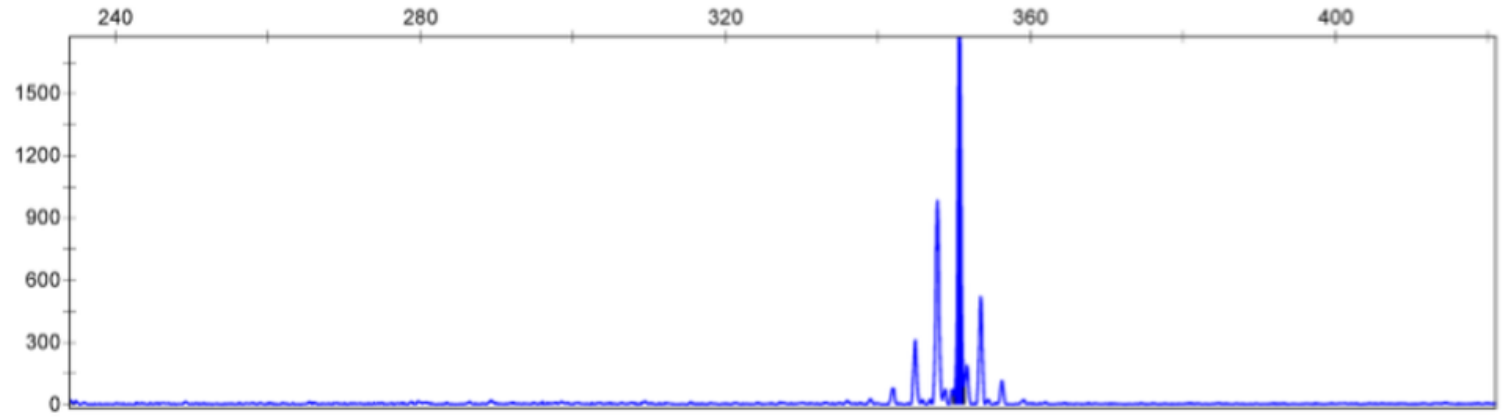

\section{Figure 2}

Three of the five clinically diagnosed patients agreed to undergo genetic testing. Among them, the number of CAG repetitions of the proband (II-3) is 48 , and the number of repetitions of the three generations of II- 3 (Figure 2.1), III-7 (Figure 2.2), and IV-6 (Figure 2.3) is 48. Consider The number of CAG repeats is stably inherited in this family. 
$(2.1)$ AR-CP21D0270(6335) 014 2019-03-01 B02.fsa AR-CP21D0270(6335) None

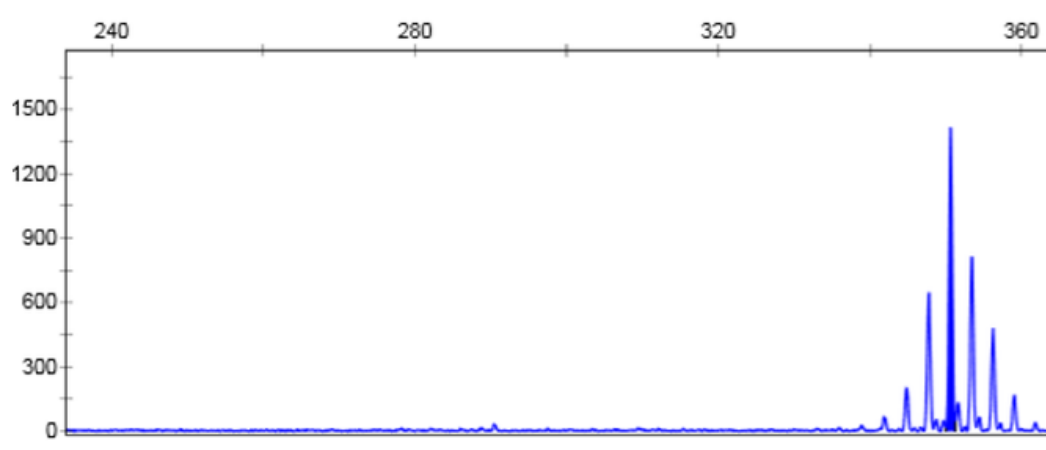

(2.2)
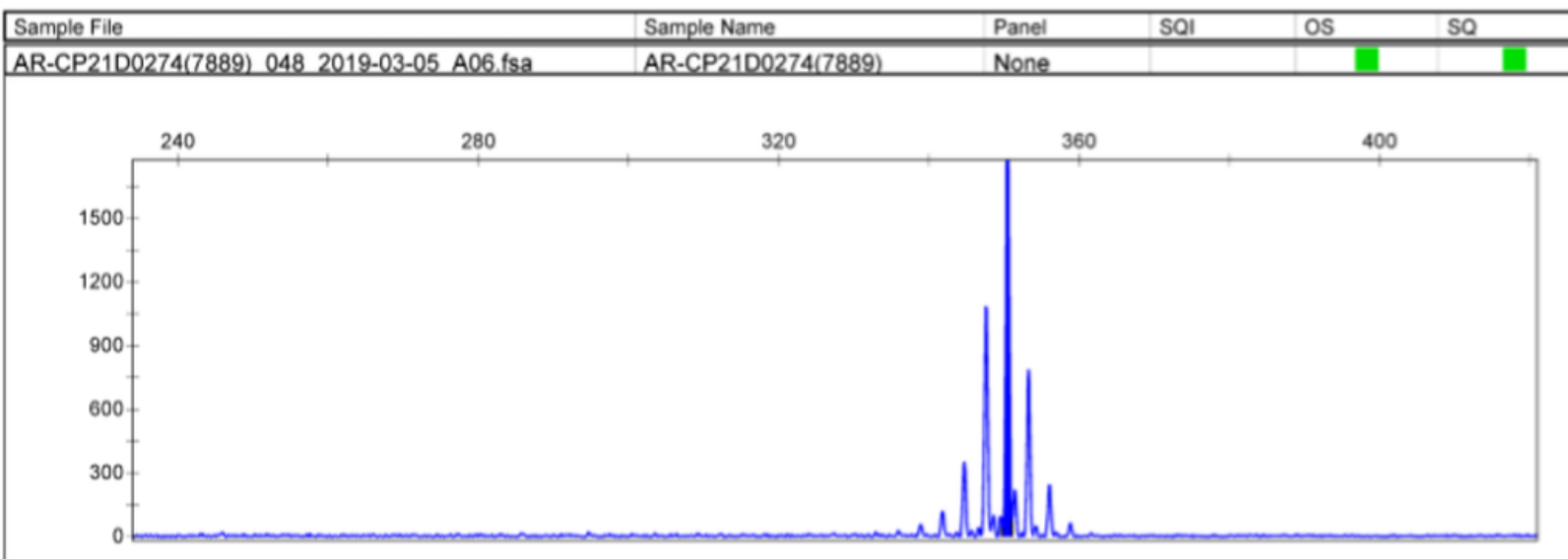

(2.3)

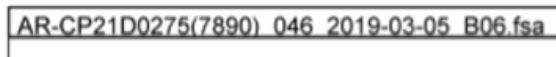
AR-CP2100275(7890) None

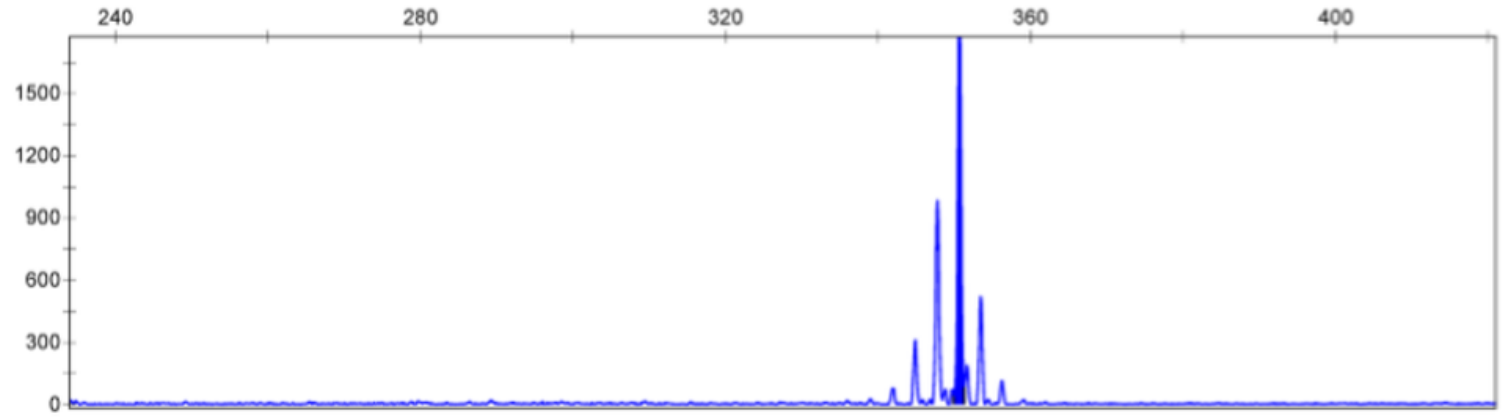

\section{Figure 2}

Three of the five clinically diagnosed patients agreed to undergo genetic testing. Among them, the number of CAG repetitions of the proband (II-3) is 48 , and the number of repetitions of the three generations of II- 3 (Figure 2.1), III-7 (Figure 2.2), and IV-6 (Figure 2.3) is 48. Consider The number of CAG repeats is stably inherited in this family. 


\section{family of Kennedy disease}

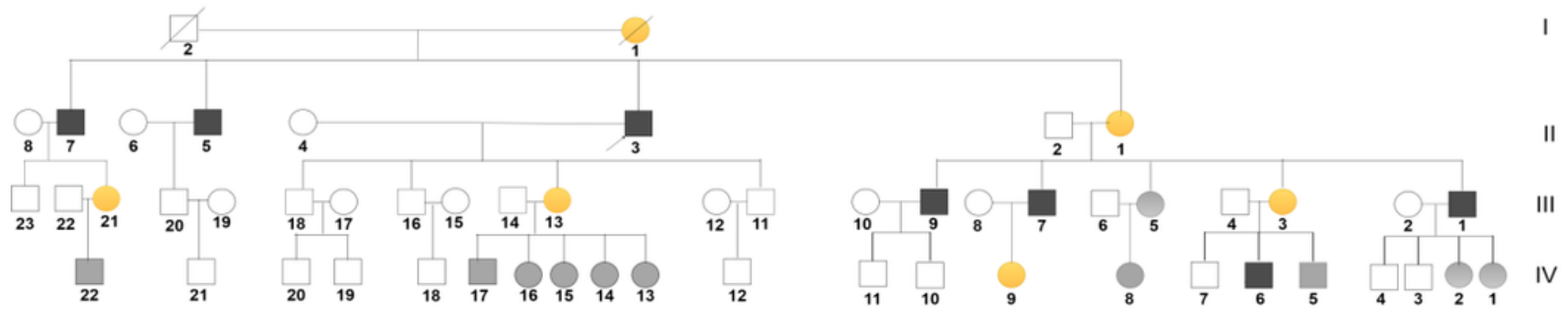

Figure 3.5

$\square$ Normal male $\square$ suspicious $\bigcirc$ Female carrier $\square$ Male patient $\bigcirc$ suspicious female carrier

\section{Figure 3}

pedigree diagram to provide a basis for genetic counseling of the family

\section{family of Kennedy disease}

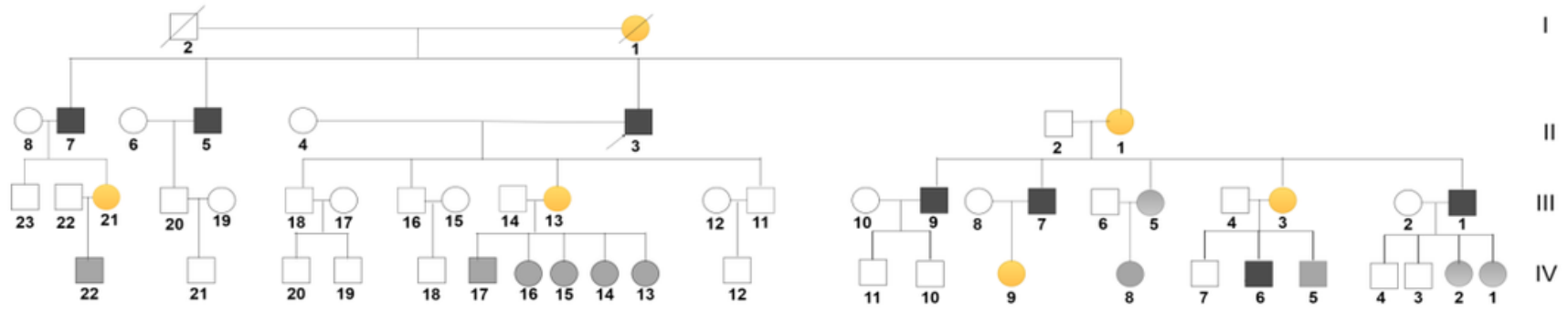

Figure 3.5

$\square$ Normal male $\square$ suspicious $\bigcirc$ Female carrier $\square$ Male patient $\bigcirc$ suspicious female carrier

Figure 3

pedigree diagram to provide a basis for genetic counseling of the family 